Thorax, 1978, 33, 511-513

\title{
Staged bilateral lobectomy for synchronous bilateral squamous cell carcinoma of lung
}

\author{
AJAIB S SOORAE \\ From the Thoracic Surgical Unit, Royal Victoria Hospital, Belfast BT 12 6BA, Northern Ireland
}

\begin{abstract}
Soorae, A S (1978). Thorax, 33, 511-513. Staged bilateral lobectomy for synchronous bilateral squamous cell carcinoma of lung. The history, diagnosis, and treatment by staged bilateral lobectomy of synchronous bilateral squamous cell carcinoma of the lung occurring in a 61-year-old man is presented. Histological diagnosis of pulmonary lesions suspected of being carcinomas should always be obtained, and extrathoracic metastasis should be excluded by all available means before planning treatment. The side showing evidence of greater malignancy on histology, or the one with the larger carcinoma, should be operated on first. Conservative, staged, bilateral resection is recommended.
\end{abstract}

Carcinoma of the lung is one of the commonest tumours in adults, but the incidence of multiple primary bronchogenic carcinoma is relatively low (Struve-Christensen, 1971). Such carcinomas can be synchronous or metachronous (Martini and Melamed, 1975). Bilateral synchronous primary carcinoma of the lung has been infrequently diagnosed in life and rarely treated successfully by resection.

\section{Case report}

In April 1977 a 61-year-old man was admitted complaining mainly of cough and haemoptysis for two months. He used to smoke at least 20 cigarettes a day but had given up smoking three years previously.

On examination his pulse was regular, and his blood pressure was $150 / 100 \mathrm{mmHg}$. Normal breath sounds were heard over both lung fields. There was no lymphadenopathy or hepatomegaly.

Full blood count, blood chemistry, and electrocardiogram were all normal. His FVC was $2 \cdot 71$ and $\mathrm{FEV}_{1} 21$, with predicted values of 3.51 and 2.71 respectively. Repeated sputum cytology showed no malignant cells. A posteroanterior chest radiograph showed bilateral rounded opacities in the mid-zones. The opacity in the right lung measured $4 \times 3 \mathrm{~cm}$ and the one in the left lung $5 \times 4 \mathrm{~cm}$ (see figure). Bronchoscopy was normal. Bilateral aspiration needle biopsies were carried out under radiographic control. The overall ap-

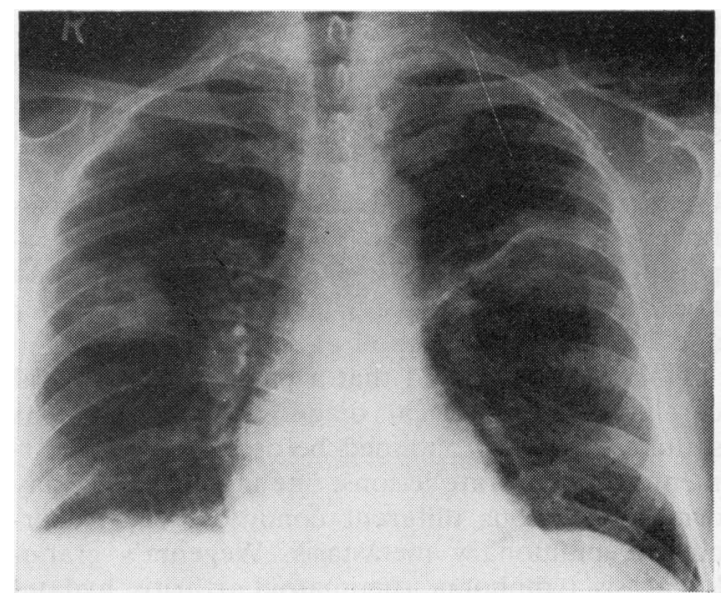

Figure Posteroanterior chest radiograph showing bilateral carcinomas.

pearance of both these biopsies was that of squamous cell carcinoma, consistent with a primary tumour.

It was decided to operate on the patient in two stages as his respiratory reserve was not adequate to withstand a synchronous bilateral thoracotomy.

At left thoracotomy, a mass, about $4 \times 4 \mathrm{~cm}$, was found in the upper lobe, and left upper lobectomy, with preservation of the lingula, was carried out. Histologically the tumour was a squamous cell carcinoma. The bronchial margin was free from tumour, and the lymph nodes were not infiltrated. 
Recovery was uneventful, and the patient was discharged on the tenth day. He was re-admitted three weeks later for a right thoracotomy.

At operation, a tumour about $3 \times 3 \mathrm{~cm}$ was found in the right upper lobe extending across the horizontal fissure to the middle lobe. Right upper lobectomy, including a wedge of the middle lobe, was caried out. Histologically, this was also a squamous cell carcinoma with pronounced giant cell proliferation. The pulmonary vessels were encircled by tumour but were not invaded by it. The middle lobe was infiltrated by tumour, which was mainly confined to the pleural surface.

Postoperative progress was complicated by sputum retention, ultimately necessitating tracheostomy. After this the patient maintained a slow but steady recovery, and he was discharged three weeks later.

Nine months later the patient was asymptomatic and was clinically free from recurrence or metastasis. His chest radiograph was satisfactory.

\section{Discussion}

Because of the relative rarity of synchronous bilateral primary carcinomas presenting as a clinical problem, and hence a lack of any large series, this subject has not received much attention. Martini and Melamed (1975) could find only four cases of bilateral synchronous carcinoma out of 5163 cases reviewed by them, while Razzuk et al (1974) reported two cases out of 2664. Many large series do not even mention double primary tumours (Walter and Pryce, 1955; Rienhoff et al, 1958).

Once it is suspected that a patient has bilateral carcinoma, histological diagnosis of the lesions should always be obtained before planning treatment. Two separate lesions, one in each lung, may occur in various different conditions like tuberculosis, pulmonary metastasis, Wegener's granulomatosis, lymphoma, rheumatoid arthritis, hydatid disease, conglomerate pneumoconiosis, mineral oil aspiration, pulmonary infarction, and several other diseases. Cases have occurred in which one side had a malignant tumour and the other a granuloma (Clifton et al, 1964). The diagnosis is easily established in centrally placed carcinomas, which are accessible through a bronchoscope. The two carcinomas were peripheral in the case reported, and we find that aspiration needle biopsy, under radiographic control, is very useful in such circumstances and carries negligible risks to the patient.

The presence of extrathoracic metastasis should be excluded by a thorough clinical examination and other available means.
Bilateral surgical resection offers the best chance of cure if the general condition of the patient per mits this. Survival after adequate resection in thise group of patients is essentially the same as in single primary lung cancer managed in a similam manner (Martini and Melamed, 1975).

Struve-Christensen (1971) recorded four pub lished cases of bilateral resection for synchronous bilateral carcinoma-three of them had a stage $\Phi$ removal (Langston and Sherrick, 1962; Payne $e \overrightarrow{\overrightarrow{2}}$ al, 1962; Struve-Christensen, 1971), and one was removed by simultaneous resection (Petersen et af 1963). Martini and Melamed (1975) reported three more patients with bilateral carcinomas who underwent staged bilateral surgical resections. The argument put forward for simultaneous bilateral resection is that it prevents the possibility of ads ditional tumour growth in the opposite lung durines the waiting period. If, on the other hand, by staged resection the operative risks can be reduced and the waiting period between the two operations kept to a minimum, then this factor of tumour growth is probably not of much importance.

The side to be operated on first should be the one in which the tumour either shows greatep evidence of malignancy or is larger in size. conservative approach to resection, consistent witlo an adequate cancer operation, should be adopted $\vec{\odot}$ A strong case can be made for elective tracheost3 omy, after the second operation, in patients who have marginal respiratory reserve and who are unable to maintain a clear airway by coughing.

I am grateful to Mr T B Smiley for allowing me to report this case and $\mathrm{Mr} \mathrm{H}$ M Stevenson for his advice and helpful criticism of the paper.

\section{References}

Clifton, E E, Gupta, T D, and Pool, J L (1964) Bilateral pulmonary resection for primary or meta static lung cancer. Cancer, 17, 86-94.

Langston, H T, and Sherrick, J C (1962). Bilateraik simultaneous bronchogenic carcinoma: Report of case of surgical excision. Journal of Thoracic and Cardiovascular Surgery, 43, 742-751.

Martini, N, and Melamed, M R (1975). Multiple primary lung cancers. Journal of Thoracic an Cardiovascular Surgery, 70, 606-612.

Payne, W S, Clagett, O T, and Harrison, E G (1962).o Surgical management of bilateral malignant lesion $\overline{-}$ of the lung. Journal of Thoracic and Cardiovascula Surgery, 43, 279-290.

Peterson, B E, Pirogov, A I, and Smulevich, V $\mathbb{B}$ (1963). Simultaneous bilateral lobectomy in a case of bilateral primary cancer of the lungs. Journal of Thoracic and Cardiovascular Surgery, 45, 705-7120 
Razzuk, M A, Pockey, M, Urschel, H C, and Paulson, D L (1974). Dual primary bronchogenic carcinoma. Annals of Thoracic Surgery, 17, 425-433.

Rienhoff, W F, King, J D B, and Dana, G W, jun (1958). Surgical treatment of carcinoma of the lung: Evaluation of 699 cases from 1933 through 1956. Journal of American Medical Association, 166, 228-232.

Struve-Christensen, E (1971). Diagnosis and treatment of bilateral primary bronchogenic carcinoma. Journal of Thoracic and Cardiovascular Surgery, 61, 501-513.

Walter, J B, and Pryce, D M (1955). The histology of lung cancer. Thorax, 10, 107-116.

Requests for reprints to: Ajaib S Soorae, FRCSEd, FRCS(Eng), Thoracic Surgical Unit, Royal Victoria Hospital, Belfast BT12 6BA, Northern Ireland. 